\title{
Tendencia de la prevalencia y factores asociados a la infección por Giardia duodenalis en escolares y preescolares de una zona rural de Cundinamarca
}

\author{
María del Pilar Chaves, Julián Alfredo Fernández, Isabel Ospina, Myriam Consuelo López, \\ Ligia Moncada, Patricia Reyes
}

Unidad de Parasitología, Departamento de Salud Pública, Facultad de Medicina, Universidad Nacional de Colombia, Bogotá D. C., Colombia

Introducción. La giardiasis continúa siendo un problema de salud pública en amplias regiones del mundo y en Colombia. Esta infección se encuentra asociada con la pobreza, el saneamiento ambiental inadecuado y la deficiencia de servicios públicos.

Objetivo. Describir la tendencia de la prevalencia de la infección por Giardia duodenalis en la población escolar y preescolar de La Virgen, Quipile, Cundinamarca, y su posible asociación con las variables socioeconómicas.

Materiales y métodos. Se tomaron tres puntos de corte realizados en los años 1995, 2001 y 2005; en cada uno se realizó muestreo no aleatorio de niños entre 0 y 15 años de edad, a quienes se les hizo examen coprológico para determinar la infección por G. duodenalis y otros parásitos intestinales. En el 2005 se realizó, además, una encuesta a los cuidadores de los sujetos de estudio sobre las condiciones sociodemográficas, económicas y las prácticas sanitarias del núcleo familiar.

Resultados. La prevalencia de la infección por G. duodenalis no ha cambiado significativamente en el periodo de estudio: se encontró $13,60 \%$ en el corte de $1995,12,84 \%$ en el 2001 y $15,16 \%$ en el 2005. Los resultados sugieren que el pertenecer al régimen subsidiado se asocia con la infección por $G$. duodenalis (razón de prevalencias, $\mathrm{RP}=4,47$; intervalo de confianza, IC95\%: 0,63-32,02), al igual que el no tener acueducto (RP=2,59; IC95\%: 1,13-5,97) y el dedicarse a la agricultura ( $\mathrm{RP}=2,53$; IC95\%:1,00-6,41).

Conclusión. La giardiasis continúa siendo un problema de salud pública en esta población; mientras persistan los factores ambientales y socioeconómicos asociados con la infección, esta protozoosis intestinal no podrá ser controlada.

Palabras clave: giardiasis/epidemiología, Giardia, prevalencia, factores de riesgo, calidad de vida, preescolar.

Giardia duodenalis prevalence and associated risk factors in preschool and school-age children of rural Colombia

Introduction. Giardia doudenalis infection remains an important public health problem worldwide, as well as in Colombia. This infection is associated with poverty and lack of public services.

Objective. The study was designed to describe prevalence trends of Giardia duodenalis infections in preschool and school-age children from the village of La Virgen, Quipile, Cundinamarca, and its possible association with socioeconomic variables.

Materials and methods. Three cross-sectional studies were undertaken in 1995, 2001 and 2005. In each, a non-random sample was selected among children aged $<1$ to 15 years. Fecal samples were collected for direct examination in order to determine G. duodenalis infection and other intestinal parasites. During the 2005 survey information was collected concerning the sociodemographic and economic conditions in each family group from which a child was sampled.

Results. The prevalence of $G$. duodenalis infection did not change significantly during the study period-13.6\% in 1995, 12.8\% in 2001 and $15.2 \%$ in 2005 . These results suggested that 
belonging to the subsidized health care regime was associated with G. duodenalis infection (prevalence rate [PR]: 4.47; 95\% confidence interval [95\% Cl] 0.6-32.0), not having water supply (PR 2.6; 95\% Cl 1.1-6.0) and involvement in agricultural activities (PR 2.5; 95\% Cl 1.06.4).

Conclusion. Giardiasis is a continuing public health problem at La Virgen. As long as the environmental and socioeconomic factors associated with infection persist, this intestinal protozoan infection will not be controlled.

Key words: Giardiasis/epidemiology, Giardia, prevalence, risk factors, quality of life, child, preschool.

La infección por Giardia duodenalis es una de las causas más frecuentes de parasitismo intestinal, especialmente en niños de uno a cinco años, mujeres embarazadas y en individuos con sida, tanto en países desarrollados como en vía de desarrollo (1-4). La infección se adquiere por vía oral al ingerir entre 10 y 100 quistes del parásito $(5,6)$. Los factores de riesgo para adquirir la infección incluyen viajes a zonas de alta prevalencia (aproximadamente, $5 \%$ de las diarreas del viajero), internación en guarderías o centros de cuidado, transmisión orofecal en homosexuales e ingestión de agua o de vegetales contaminados con quistes (6).

El $60 \%$ de los individuos infectados son asintomáticos (3), aunque también puede ocasionar cuadros clínicos variados que van desde síntomas inespecíficos, diarrea aguda o crónica, dolor abdominal, meteorismo, flatulencia, presencia de moco en la deposición y, ocasionalmente, fiebre, hasta síndrome de mala absorción intestinal cuando hay una carga parasitaria alta (7).

Dependiendo de la presencia de los factores de riesgo mencionados anteriormente para adquirir el parásito y de las condiciones de salubridad de la población, la presentación de la infección varía de menos de $1 \%$ a $30 \%(2,3,7)$. En Colombia, según las dos encuestas nacionales de morbilidad realizadas en 1965 y 1980, la prevalencia general de Giardia duodenalis hallada en los niños entre cinco y 14 años fue de $14,6 \%$ y $21,7 \%$,

\footnotetext{
Correspondencia:

Patricia Reyes, Unidad de Parasitología, Departamento de Salud Pública, Facultad de Medicina, Universidad Nacional de Colombia, Ciudad Universitaria, carrera 30 calle 45, Bogotá, D. C., Colombia.

Teléfono: (571) 3165505

preyesh@unal.edu.co
}

Recibido: 20/02/07; aceptado: 24/04/07 respectivamente; también se observó un incremento de $3,7 \%$ en los menores de cuatro años, en los cuales la prevalencia pasó de 27,4\% en 1965 a 31,1\% en 1980. Para los departamentos de la Región Central entre los que se encuentra Cundinamarca, las prevalencias reportadas en 1965 fueron de $24,6 \%$ para los niños entre uno y cuatro años y de $9,4 \%$ para el grupo de edad entre cinco y 14 años. Al comparar estas prevalencias con las del año de 1980 para los mismos grupos de edad se observó un incremento de $7,6 \%$ y $12 \%$ respectivamente. Las prevalencias del parásito informadas en la población general de la Región Central fueron de 10,5\% en el año 1965 y 13,3\% en 1980 (8). Al comparar los datos de los dos años se observa que hubo un incremento tanto en la población general como en la población infantil, de igual manera se observó que al comparar las prevalencias de Giardia duodenalis en las cinco zonas estudiadas: Región Atlántica, Región Pacífica, Región Central, Región Oriental y el Distrito Capital hubo un aumento de la prevalencia a excepción del Distrito Capital. En un estudio reciente realizado en Armenia, en niños de 1a 7 años atendidos en los hogares de madres comunitarias del Instituto Colombiano de Bienestar Familiar (ICBF), se informó una prevalencia de $12,8 \%$ (9).

Varios autores han asociado en diversos estudios, la infección por $G$. duodenalis con condiciones socioeconómicas desfavorables y con malas prácticas de higiene (10-15). La falta de acueducto y alcantarillado $(10,11)$, el bajo nivel educativo de las madres y los bajos ingresos de los padres $(12,13)$ fueron identificados como factores de riesgo para contraer la infección por $G$. duodenalis.

La presente investigación se planteó con el fin de describir la tendencia de la prevalencia y de los factores asociados a la infección por $G$. duodenalis 
en niños escolares y preescolares de la población de La Virgen, Quipile, Cundinamarca, dado que este agente es el principal protozoario patógeno que afecta a este tipo de población, rural y empobrecida, con un alto impacto sobre la salud colectiva.

\section{Materiales y métodos}

El área de estudio fue la población de La Virgen, inspección del municipio de Quipile (Cundinamarca), ubicada a $100 \mathrm{~km}$, aproximadamente, al suroccidente de Bogotá (16).

Para el estudio de la tendencia de la prevalencia de la infección por $G$. duodenalis se tomaron los datos de tres estudios de corte transversal realizados, el primero, en 1995, incluyó 272 niños entre 2 y 15 años de edad (edad media de siete años), el segundo, en el 2001, contó con 288 sujetos entre 0 y 15 años (edad media de 6 años) y, el último, en el 2005, con 213 sujetos entre 1 y 15 años de edad (edad media de ocho años). En cada corte se realizó un muestreo por conveniencia de niños preescolares y escolares, mediante una convocatoria abierta en la cabecera municipal en la que se invitó a la comunidad a participar en el estudio y a la cual asistieron voluntariamente los niños con sus padres; todos los asistentes en cada punto de corte fueron incluidos en el estudio.

Para determinar la infección por parásitos intestinales, se tomaron muestras de materia fecal a todos los sujetos de estudio. Las muestras se conservaron en formol al $10 \%$ y se transportaron al Laboratorio de Parasitología de la Facultad de Medicina de la Universidad Nacional de Colombia, donde fueron analizadas exclusivamente por el método de concentración de Ritchie-Frick modificado para la determinación de la infección por $G$. duodenalis y otros parásitos intestinales (17).

Se observó la tendencia de la giardiasis estratificada por sexo y edad. Todos los niños en los que se detectó infección por $G$. duodenalis u otros parásitos intestinales recibieron tratamiento según los esquemas vigentes (18-20).

Además, en el 2005, se realizó una encuesta para evaluar las principales condiciones socioeconó- micas y las prácticas sanitarias asociadas con parasitismo intestinal.

La encuesta la respondieron los responsables del menor. El informante no fue seleccionado por los investigadores y asistió voluntariamente. La relación entre el informante y el sujeto de estudio era variable. Fueron entrevistados 131 responsables de los niños: $98(75,4 \%)$ eran madres, 9 padres $(6,9 \%)$ y $23(17,7 \%)$ tenían otra relación diferente con los sujetos de estudio.

Los investigadores que analizaron las muestras no conocían el estado clínico de los sujetos de estudio y los encuestadores en ningún caso conocían el diagnóstico parasitológico. En el estudio analítico se incluyeron 131 sujetos de quienes se disponía de la medición de la infección parasitaria y de las variables de interés.

Se exploró la asociación entre las variables demográficas y se estableció una estratificación de la exposición para cada caso.

Se tomó como población de referencia aquélla que por definición debería tener una menor prevalencia. Se estimaron las razones de prevalencia y sus respectivos intervalos de confianza de $95 \%$ (IC95\%) para cada asociación, mediante el paquete Epi-info V. Se analizaron los resultados y se discutió el papel del error.

Aspectos éticos. El presente estudio contó con la aprobación del Comité de Ética de la Facultad de Medicina de la Universidad Nacional de Colombia. La identidad de los sujetos de estudio se mantiene en confidencialidad.

\section{Resultados}

Tendencia de la prevalencia de infección por G. duodenalis. La prevalencia de $G$. duodenalis en la población total de estudio fue de $13,60 \%$ en el corte de $1995,12,84 \%$ en el 2001 y $15,16 \%$ en el 2005 (cuadro 1).

La observación estratificada por edad no muestra diferencias significativas en las prevalencias según la edad. En 1995 y 2001, éstas fueron ligeramente mayores en los menores de cinco años comparadas con el grupo de edad entre 5 y 15 años, mientras que, en el corte del 2005, se encontró una prevalencia de sólo $3,4 \%$ para los 
Cuadro 1. Distribución por grupos de edad de la tendencia de la prevalencia de la infección por G. duodenalis en La Virgen, Quipile, Cundinamarca.

\begin{tabular}{|c|c|c|c|c|c|c|c|c|c|}
\hline \multirow[b]{2}{*}{ Edad (años) } & \multicolumn{3}{|c|}{1995} & \multicolumn{3}{|c|}{2001} & \multicolumn{3}{|c|}{2005} \\
\hline & $\mathbf{n}$ & $\mathbf{p}$ & pr (\%) & $\mathbf{n}$ & $\mathbf{p}$ & pr (\%) & $\mathbf{n}$ & $\mathbf{p}$ & $\operatorname{pr}(\%)$ \\
\hline$<5$ & 7 & 48 & 14,58 & 10 & 72 & 13,88 & 1 & 29 & 3,4 \\
\hline $5-15$ & 30 & 224 & 13,39 & 27 & 216 & 12,5 & 31 & 182 & 17 \\
\hline Total & 37 & 272 & 13,60 & 37 & 288 & 12,84 & 32 & 211 & 15,16 \\
\hline
\end{tabular}

$\mathrm{n}$ : número de casos; p: población examinada; pr: prevalencia

menores de cinco años; sin embargo, en este punto de corte la muestra fue muy pequeña $(p=29)$.

Factores socioeconómicos asociados con Giardia. Se exploró la asociación de los principales indicadores de la condición socioeconómica con la infección por $\mathbf{G}$. duodenalis (cuadro 2).

Los resultados sugieren una asociación positiva de la infección por $G$. duodenalis con pertenecer al régimen subsidiado o ser vinculado $(R P=4,47$; IC95\%: 0,63-32,02), con la ausencia de acueducto (RP=2,59; IC95\%: 1,13-5,97) y con la práctica de la agricultura por parte de los cuidadores ( $R P=2,53$; IC95\%: 1,00-6,41). Sin embargo, sólo los intervalos de confianza de las dos últimas variables no incluyeron la hipótesis nula. Las asociaciones entre la giardiasis y el no tener casa propia (RP=1,11; IC95\%: 0,48-2,57) y el uso como fuente principal de agua la de los ríos, quebradas o pozos profundos ( $\mathrm{RP}=1,84$; IC95\%: 0,70-4,84) fueron débiles y en ambos casos el intervalo de confianza incluyó la hipótesis nula.

\section{Discusión}

La observación de la prevalencia de la infección por G. duodenalis en este trabajo no mostró cambios significativos durante el periodo de estudio, lo cual sugiere que en esta población persisten los factores ambientales y las malas prácticas sanitarias asociadas a la infección. En el país, según los datos disponibles de la Encuesta Nacional de Salud, entre 1965 y 1980, el parasitismo por $G$. duodenalis tuvo un incremento de $3,7 \%$ en los menores de cinco años y de $7,1 \%$ en niños entre 5 y 14 años (8).

En este estudio no se encontraron diferencias significativas en la distribución de la infección por
G. duodenalis por edad, excepto para el año 2005, en el que la prevalencia de esta infección se redujo cuatro veces en el grupo de menores de 5 cinco años; sin embargo, esa diferencia puede ser explicada porque en este año se contó con una muestra más pequeña que en los otros puntos de corte. En contraste, otros autores demuestran una mayor distribución de casos entre los menores de cinco años $(14,15,21)$. La encuesta de 1980 mostró también una mayor prevalencia en los preescolares, $31,1 \%$, que en los niños entre 5 y 14 años de edad, $21,7 \%$ (8).

Es importante mencionar que una limitante del análisis de los cambios en las prevalencias de giardiasis en los menores de cinco años es que, como se refirió en la metodología, los rangos de edad de las muestras en cada punto de corte no son exactamente los mismos, por lo que las prevalencias en cada corte para este grupo de edad pueden no ser comparables entre ellas.

Entre los factores socioeconómicos analizados, aquéllos que sugieren una asociación con la giardiasis son: pertenecer al régimen subsidiado, no tener casa propia, ni acueducto; tener como fuente principal agua de pozos o ríos y la práctica de la agricultura por parte de los cuidadores de los niños. Sin embargo, la mayoría de los intervalos de confianza de las razones de prevalencia para las asociaciones estudiadas en este trabajo son muy amplios e incluyen, además, a la hipótesis nula, lo que indica que estos resultados podrían explicarse por el error aleatorio. Esta limitante se explica principalmente por el reducido tamaño de la muestra.

En este estudio no se encontró asociación entre la escolaridad de los padres y la infección por $G$. 
Cuadro 2. Asociación de variables socioeconómicas y sanitarias con la infección por G. duodenalis en La Virgen, Quipile, Cundinamarca, 2005.

\begin{tabular}{|c|c|c|c|c|c|c|c|}
\hline \multirow{2}{*}{$\begin{array}{l}\text { Variables de riesgo } \\
\text { Escolaridad del padre encuestado }\end{array}$} & \multirow[t]{2}{*}{ Total } & \multicolumn{2}{|c|}{$\begin{array}{r}\text { Infecc } \\
\text { Positivo } \\
\text { N (\%) }\end{array}$} & \multicolumn{2}{|c|}{$\begin{array}{c}\text { Giardia } \\
\text { Negativo } \\
\text { N ( \% ) }\end{array}$} & \multicolumn{2}{|c|}{$\begin{array}{l}\text { Razón de prevalencia } \\
\text { (IC95\%) }\end{array}$} \\
\hline & & & & & & & \\
\hline$\leq$ Primaria & 84 & 12 & $(14,3)$ & 72 & $(85,7)$ & 0,94 & $(0,40-2,22)$ \\
\hline $\begin{array}{l}>\text { Primaria } \\
\text { Servicio de salud }\end{array}$ & 46 & 7 & $(15,2)$ & 39 & $(84,8)$ & 1 & \\
\hline Régimen subsidiado y vinculados & 95 & 17 & $(17,9)$ & 78 & $(82,1)$ & 4,47 & $(0,63-32,02)$ \\
\hline Régimen contributivo & 25 & 1 & (4) & 24 & $(96)$ & 1 & \\
\hline \multicolumn{8}{|l|}{ Práctica de la agricultura } \\
\hline Sí & 12 & 4 & $(33,3)$ & 8 & $(6,7)$ & 2,53 & $(1,00-6,41)$ \\
\hline No & 114 & 1 & $(0,9)$ & 113 & $(99,1)$ & 1 & \\
\hline \multicolumn{8}{|l|}{ Carencia de casa propia } \\
\hline Sí & 72 & 11 & $(15,3)$ & 61 & $(84,7)$ & 1,11 & $(0,48-2,57)$ \\
\hline No & 58 & 8 & $(13,8)$ & 50 & $(86,2)$ & 1 & \\
\hline \multicolumn{8}{|l|}{ Piso de tierra en la vivienda } \\
\hline Sí & 100 & 13 & $(13)$ & 8 & $(87)$ & 0,65 & $(0,27-1,56)$ \\
\hline No & 30 & 6 & (20) & 24 & $(80)$ & 1 & \\
\hline \multicolumn{8}{|l|}{ Presencia de acueducto } \\
\hline No & 44 & 11 & $(25)$ & 33 & (75) & 2,59 & $(1,13-5,97)$ \\
\hline Sí & 83 & 8 & $(9,6)$ & 75 & $(90,4)$ & 1 & \\
\hline \multicolumn{8}{|l|}{ Fuente principal de agua } \\
\hline Quebrada, río o pozo & 75 & 13 & $(17,3)$ & 62 & $(82,7)$ & 1,84 & $(0,70-4,84)$ \\
\hline Acueducto veredal & 53 & 5 & $(9,4)$ & 48 & $(90,6)$ & 1 & \\
\hline \multicolumn{8}{|l|}{ Presencia de alcantarillado } \\
\hline No & 115 & 17 & $(14,8)$ & 98 & $(85,2)$ & 0,81 & $(0,22-3,07)$ \\
\hline Sí & 11 & 2 & $(18,2)$ & 9 & $(81,8)$ & 1 & \\
\hline
\end{tabular}

IC: intervalo de confianza del $95 \%$

duodenalis, lo que contrasta con los resultados de otros autores. Por ejemplo, Machado et al., en San Pablo, Brasil, registraron que los preescolares y escolares hijos de padres con baja escolaridad $\left(\chi^{2}=59,79 ; p<0,01\right)$ y bajo nivel de ingresos $\left(\chi^{2}=35,32 ; p<0,01\right)$ tenían un mayor riesgo de infección por G. duodenalis (12). Este mismo hallazgo lo informan Quihui et al., en 507 niños de tres comunidades rurales de México, con una fuerza de asociación alta con el bajo nivel de escolaridad (razón de momios, RM=6,0; IC95\%: 1,6-22,6), con los bajos ingresos ( $\mathrm{RM}=4,5$; IC95\%: $2,5-8,2)$ y con el desempleo de los padres ( $R M=3,3$; IC95\%: 1,5-7,4) (13). El hecho de no encontrarse esta asociación en este estudio podría explicarse porque la mayoría de los individuos entrevistados tenían baja escolaridad y no fue posible seleccionar un grupo más grande de padres con escolaridad alta para explorar esta asociación.

Estar afiliado al régimen subsidiado o estar vinculado, en comparación con pertenecer al régimen contributivo, se asoció con la infección por G. duodenalis, lo cual podría explicarse por las diferencias en el acceso y la calidad de servicios de salud. Vinculados y subsidiados son atendidos en las Empresas Sociales del Estado, mientras que los afiliados al régimen contributivo tienen, además, disponibilidad de acceso a otras instituciones de salud. Esta asociación también podría explicarse porque vinculados y subsidiados comparten condiciones económicas (bajo nivel de ingresos, baja escolaridad) que aumentan el riesgo de infección por $G$. duodenalis.

No tener casa propia puede considerarse un indicador indirecto de condiciones económicas precarias de una familia. Por tanto, se esperaría que el riesgo de infección por $G$. duodenalis fuera mayor en quienes carecen de vivienda propia en comparación con quienes la poseen, como se ha encontrado en otras investigaciones $(12,14)$. El no haber encontrado esta asociación en este estudio se explica posiblemente por el hecho de 
que en La Virgen no existen grandes diferencias entre las condiciones de las viviendas habitadas por sus propietarios y aquéllas de las que están ocupadas en arriendo.

El no tener como fuente principal de agua para consumo un acueducto sino la obtenida de quebradas, ríos o pozos profundos, se ha reportado como factor de riesgo por otros autores $(10,14)$ y se explica, principalmente, por la presencia de una mayor concentración de quistes de este protozoario en las aguas no tratadas adecuadamente. Esta asociación también se encontró en este estudio, a pesar de que el acueducto veredal de La Virgen no implica tratamiento del agua sino solamente su transporte.

Al parecer los hogares agricultores están más expuestos a los componentes de las aguas escasamente tratadas o sin tratar y, en consecuencia, a una mayor concentración de quistes, que quienes se dedican a otro tipo de labores. También, los agricultores se exponen a la contaminación de los suelos en los cuales trabajan. En Pakistán, un estudio de casos y controles realizado en una población cuya economía gira entorno al trabajo textil y a la agricultura, mostró que los hogares dedicados a este último oficio, que usaban aguas residuales sin tratar para irrigar sus cultivos, tenían riesgo incrementado de infección por $G$. duodenalis ( $R M=3,3$; IC95\%: 2,5-4,4) (10). Ser agricultor, también se encontró como factor de riesgo en un estudio hecho en Marruecos, en 214 personas (riesgo relativo $=4,3)(11)$.

Aunque en la mayoría de casos fue la madre quien respondió la encuesta, también se entrevistaron padres, abuelos e, incluso, cuidadores sin parentesco, lo que pudo causar un sesgo de información de tipo memoria selectiva, ya que algunos informantes, como, por ejemplo, las madres, comparadas con los cuidadores sin parentesco, podrían recordar mejor la exposición o tener diferentes grados de interés en contestar verazmente la encuesta.

Sin embargo, la influencia del sesgo de información diferencial se minimizó porque los encuestadores no conocían el diagnóstico parasitológico de los sujetos de estudio y, de la misma manera, los investigadores que realizaron el examen coprológico eran ciegos a los resultados de la encuesta y al estado clínico de los niños.

La muestra utilizada en todos los puntos de corte se seleccionó mediante convocatoria abierta en la cabecera de la inspección de La Virgen, lo que pudo generar una barrera geográfica para la participación en el estudio. Además, existió autoselección de los participantes, por lo que estos pudieron tener diferente probabilidad de tener la exposición o la infección que el resto de la población y las prevalencias encontradas podrían no ser extrapolables a toda la población del municipio.

A pesar de la influencia del error, se puede concluir que la infección por $G$. duodenalis continúa siendo un problema de salud pública en la inspección de La Virgen y está asociada con la pobreza, con la deficiencia en los servicios públicos y con la práctica de la agricultura. Por lo anterior, se puede asegurar que mientras persistan los factores ambientales y las condiciones socioeconómicas, se mantendrán estas prevalencias de infección por G. duodenalisen esta población. El mejoramiento general del nivel de calidad de vida y el saneamiento ambiental permitirán contribuir al control de la infección por $G$. duodenalis en La Virgen.

\section{Agradecimientos}

A toda la población de La Virgen, Quipile, Cundinamarca, en especial a la Hermana María Teresa Guzmán y a Tobías Castiblanco. A los auxiliares del Laboratorio de Parasitología, a las estudiantes de bacteriología del Colegio Mayor de Cundinamarca, a Olga Lucía Morales y a María del Pilar Díaz. A Angélica Knudson y a Mauricio Restrepo por sus valiosas enseñanzas y su gran esfuerzo para despertar el espíritu científico en la juventud.

\section{Financiación}

La investigación fue financiada por la División de Investigación de la Universidad Nacional de Colombia, sede Bogotá.

\section{Conflictos de interés}

Los autores declaran que durante la realización de este trabajo no incurrieron en conflicto de interés alguno. 


\section{Referencias}

1. Núñez FA, López JL, De la Cruz AM, Finlay CM. Factores de riesgo de la infección por Giardia lamblia en niños de guarderías infantiles de Ciudad de La Habana, Cuba. Cad Saúde Pública. 2003;19:109-18.

2. Adam RD. The biology of Giardia spp. Microbiol Rev. 1991;55:706-32.

3. Flanagan PA. Giardia-diagnosis, clinical course and epidemiology. A review. Epidemiol Infect. 1992;109:1-22.

4. Savioli L, Smith H, Thompson A. Giardia and Cryptosporidium join the "Neglected Diseases Initiative". Trends Parasitol. 2006;22:203-8.

5. Roxstrom-Lindquist K, Palm D, Reiner D, Ringqvist E, Svard SG. Giardia immunity-an update. Trends Parasitol. 2006;22:26-31.

6. Hill DR. Giardiasis. Issues in diagnosis and management. Infect Dis Clin North Am. 1993;7:503-25.

7. Ortega YR, Adam RD. Giardia: Overview and update. Clin Infect Dis. 1997;25:545-9.

8. Corredor A, Arciniegas E, Hernández CA. Parasitismo intestinal, Santa Fe de Bogotá, D.C.: Instituto Nacional de Salud; 2000.

9. Giraldo-Gómez JM, Lora F, Henao LH, Mejía S; Gómez-Marín JE. Prevalencia de giardiasis y parásitos intestinales en preescolares de hogares atendidos en un programa estatal en Armenia, Colombia. Rev Salud Pública. 2005;7:327-38.

10. Ensink JH, van-der-Hoek W, Amerasinghe FP. Giardia duodenalis infection and wastewater irrigation in Pakistan. Trans R Soc Trop Med Hyg. 2006;100:538-42.

11. El Kettani S, Azzouzi EM, Maata A. Prevalence of Giardia intestinalis in a farming population using sewage water in agriculture, Settat, Morocco. Med Mal Infect. 2006;36:322-8.

12. Machado RC, Marcari EL, de Cristante S, Crisante V, Carareto CM. Giardíase e helmintíases em crianças de creches e escolas de $1^{\circ}$ e $2^{\circ}$ graus (públicas e privadas) da cidade de Mirassol (SP, Brasil). Rev Soc Bras Med Trop. 1999;32:697-704.

13. Quihui L, Valencia ME, Crompton DW, Phillips S, Hagan P, Morales G et al. Role of the employment status and education of mothers in the prevalence of intestinal parasitic infections in Mexican rural schoolchildren. BMC Public Health. 2006;6:225.

14. Laupland KB, Church DL. Population-based laboratory surveillance for Giardia sp. and Cryptosporidium $\mathrm{sp}$. infections in a large Canadian health region. BMC Infect Dis. 2005;5:72.

15. Cifuentes E, Suárez L, Espinosa M, Juárez-Figueroa L, Martínez-Palomo A. Risk of Giardia intestinalis infection in children from an artificially recharged groundwater area in Mexico city. Am J Trop Med Hyg. 2004;71:65-70.

16. Reyes P, Agudelo C, Moncada L, Cáceres E, López C, Corredor A et al. Desparasitación masiva, estado nutricional y capacidad de aprendizaje en escolares de una comunidad rural. Rev Salud Pública. 1999;1:255-64.

17. World Health Organization. Bench aids for the diagnosis of intestinal parasites. Geneve: WHO; 1992.

18. Guyatt HL. Mass chemotherapy and school-based anthelmintic delivery. Trans Roy Soc Trop Med Hyg. 1999;93:12-3.

19. Matijasevic EA. Amibiasis. Espectro clínico y tratamiento. Tribuna Med. 1995;91:290-304.

20. Gardner TB, Hill DR. Treatment of giardiasis. Clin Microbiol Rev. 2001;14:114-28.

21. Guimarães S, Sogayar MI. Occurrence of Giardia lamblia in children of municipal day-care centers from Botucatu, Sao Paulo State, Brazil. Rev Inst Med Trop Sao Paulo. 1995;37:501-6. 